\title{
Compartment and Sciatic Nerve Block for Knee Arthroplasty
}

\author{
Samir Mohamed Yassin El-Habashy, Mostafa Abd-Elhamid Hassan Abo-El Enin, Amgad \\ Zakaria El Khiat, Ayman Mahmoud Mohamed
}

Department of Anesthesiology and Intensive Care, Faculty of Medicine - Al-Azhar University

Corresponding author: Ayman Mahmoud Mohamed, email: hossamesmail31@gmail.com

\begin{abstract}
Background: Ambulatory knee procedures have become increasingly common due to the effectiveness of the anesthetic techniques that facilitate rapid and safe discharge.

Objective: To compare between spinal anesthesia versus ultrasound guided combined fascia iliaca compartment and sciatic nerve block for knee arthroplasty.

Patients and Methods: This study enrolled 40 patients (ASA) physical status (1, 2, 3), 20 patients in each group scheduled to undergo elective unilateral knee arthroplasty procedures. All patients of block group (group B) received premedication with $0.05 \mathrm{mg} / \mathrm{kg}$ midazolam intravenous injection. Group (S) (20) patients received spinal anesthesia attained by $20 \mathrm{mg} / 4 \mathrm{ml}$ of $0.5 \%$ heavy bupivacaine together with fentanyl $25 \mu$ g injected aseptically using a 22-G needle in subarachnoid space at the L4-5 level then the patients were required to wait in the lateral decubitus position on operated side for 5 minutes. Group (B) (20) patients received ultrasound guided combined fascia iliaca compartment and sciatic nerve block using a $22-\mathrm{G}$ needle.

Results: In the present study, sufficient block could not be obtained in two patients in block group, they shifted to general anesthesia and excluded from the evaluation, and we replaced them by two other patients.

Conclusion: The preferential selection and successful use of anesthesia techniques are based on not only having a short preparation time and rapid onset of action, but also on providing lower rate of complications and adverse events, a longer duration of analgesia, good patient satisfaction and optimal condition for patient discharge compared with other available agents.
\end{abstract}

Keywords: Visual analogue scale, surgical anesthesia time, Total-knee joint arthroplasty

\section{INTRODUCTION}

Ambulatory orthopaedic procedures have become increasingly common due to the effectiveness of the anesthetic techniques that facilitate rapid and safe discharge ${ }^{(I)}$.

Regional anesthesia techniques are used as an alternative to general anesthesia in these procedures. It is generally accepted that both peripheral nerve blocks and spinal anesthesia provide sufficient anesthesia, better postoperative analgesia and satisfaction than general anesthesia, in addition to being minimally invasive and using less resources ${ }^{(2)}$. Spinal anesthesia is particularly preferred by patients undergoing unilateral lower limb surgery due to the fact that only the desired region undergoes nerve blockade, which results in early mobilization and good patient satisfaction ${ }^{(3)}$.

These patients are also poor candidates for general anesthesia because they have diseases such as diabetes and hypertension and a decreased systemic functional reserve ${ }^{(4)}$.
Anatomical deformities in the aforementioned patients also reduce the success of regional anesthesia ${ }^{(5)}$.

Sudden hemodynamic changes during spinal anesthesia pose an additional risk due to the presence of accompanying diseases and previous neurological disorders ${ }^{(4)}$.

Spinal anesthesia is widely used for lower limb surgery in the elderly for efficacy, rapidity, minimal effect on mental status, reduction of blood loss, and protection against thrombo-embolic complications. But risk of severe and prolonged hypotension is associated with spinal anesthesia. This is due to the rapid extension of the sympathetic block, hindering cardiovascular adaptation and causing significant morbidity and mortality ${ }^{(6)}$.

As quadruple nerve blocks of the lower extremities require high-dose local anesthetics, the risk of systemic side effects is increased. Nevertheless, these blocks are preferred for analgesia. The use of ultrasonography (USG) 
during peripheral blocks increases their effectiveness and decreases the amount of anesthetics required ${ }^{(7)}$.

The selection of the anesthetic technique and the anesthetic agents are important as the adverse events and complications affect discharge time in ambulatory cases ${ }^{(8)}$.

\section{AIM OF THE WORK}

The aim of the present study is to compare between spinal anesthesia versus ultrasound guided combined fascia iliaca compartment and sciatic nerve block for knee arthroplasty.

\section{PATIENTS AND METHODS}

Study settings: The study was carried out in AL-Azhar University Hospitals. The study was approved by the Ethics Board of Al-Azhar University.

Inclusion criteria: After obtaining the Research/Ethics Committee approval and informed written consent from 40 patients who had the following criteria:- ASA physical status: $(1,2,3)$ with pre-existing medical condition. Both sexes (male and female). Aged from 40 to 70 years. Patient height $150-185 \mathrm{~cm}$. Patient weight 50-120 kg. Undergoing unilateral knee arthroplasty.

Exclusion criteria: Patient refusal and uncooperative patients. Patient with history of allergic reaction to local anesthetics or opioids. Presence of preoperative hypovolemia. Contraindications to neuraxial anesthesia.

\section{Methods of randomization}

Randomization of patients was done using a computerized program (SPSS). The number of cases included in this study was simple randomly allocated into two groups (20 in each group).

\section{Study groups:}

This study was conducted on 40 patients. They were classified into two groups: Group $S$ (Spinal group): included 20 patients, a 22-G needle in subarachnoid space at the L45 levels. Group B (Block group): included 20 patients, undergoing ultrasound guided combined fascia iliaca compartment and sciatic nerve block.

Anesthesia technique: All patients were requested to be fasting for $8 \mathrm{~h}$. before operation. Spinal anesthesia and block technique were explained to them preoperatively.

Patient monitoring: Pulse oximetry. ECG. Non-invasive blood pressure monitoring.

Premedication: All patients of block group were pre-medicated with intravenous midazolam $(0.05 \mathrm{mg} / \mathrm{kg}) 10 \mathrm{~min}$. before the block.

Induction: Group S (Spinal group): in this group, spinal anesthesia was attained with $20 \mathrm{mg} / 4 \mathrm{ml}$ of $0.5 \%$ heavy bupivacaine together with fentanyl $25 \mu \mathrm{g}$ injected aseptically using a 22-G needle in subarachnoid space at the L4-5 levels then the patients were required to wait in the lateral decubitus position on operated side for 5 minutes. Group B (block group): this group were administered fascia iliaca compartment and sciatic nerve block using ultrasonography, (25-G Braun needle, Melsungen, Germany). For the sciatic nerve block, $62.5 \mathrm{mg} / 12.5 \mathrm{ml}$ of $0.5 \%$ bupivacaine were mixed with $100 \mathrm{mg} / 10 \mathrm{ml}$ of $1 \%$ lidocaine. For fascia iliaca compartment block, $37.5 \mathrm{mg}$ of $0.5 \%$ bupivacaine $7.5 \mathrm{ml}$ were added $100 \mathrm{mg} / 10 \mathrm{ml}$ of $1 \%$ lidocaine.

\section{Collected data:}

Demographic and clinical data: including age, sex, weight, height, ASA physical status and duration of surgery were recorded.

Vital data: including heart rate, arterial blood pressure (systolic, diastolic and MAP), oxygen saturation and respiratory rate were recorded at base of beginning of surgery, 5, 10, 15, 20, 30 minutes, 1, 1.30, 2,4, 6,12, 18, 24 hours.

Onset time of injection (preparation time): time from skin preparation of the application site to end of local anesthetics injection was recorded.

Sensory block: Upper level of sensory block assessed by pinprick test using 22 gauge blunted needle in the midline and the highest dermatomal level of sensory block was recorded.

\section{Statistical Analysis}

Data were collected, revised, coded and entered to the Statistical Package for Social Science (SPSS) version 23 and the following were done:

Qualitative data were presented as number and percentages while quantitative data were presented as mean, standard deviations and ranges. 
The comparison between two groups with qualitative data were done by using Chisquare test and/or Fisher exact test was used instead of Chi-square test when the expected count in any cell was found less than 5 .

The comparison between two independent groups with quantitative data and parametric distribution was done by using Independent t-test.

The comparison between more than two groups with parametric distribution were done by using One Way Analysis of Variance (ANOVA).
Pearson correlation coefficients were used to assess the relation between two studied parameters in the same group.

Receiver operating characteristic curve was used to assess the best cut off point with its sensitivity, specificity, positive predictive value and negative predictive value.

The confidence interval was set to $95 \%$ and the margin of error accepted was set to $5 \%$. So, the $\mathrm{p}$-value was considered \pm significant as the following: $\mathrm{P}>0.05$ : Non significant. $\mathrm{P}<$ 0.05: Significant. $P<0.01$ : Highly significant.

RESULTS

Table (1): Difference between group (S) and group (B) regarding mean arterial blood pressure

\begin{tabular}{|c|c|c|c|c|c|c|}
\hline \multirow{2}{*}{\multicolumn{2}{|c|}{ As regard mean arterial blood pressure }} & \multirow{2}{*}{$\begin{array}{l}\text { Group S } \\
\text { No. }=20 \\
\end{array}$} & \multirow{2}{*}{$\begin{array}{l}\text { Group B } \\
\text { No. }=20\end{array}$} & \multirow{2}{*}{ Test value ${ }^{\bullet}$} & \multirow{2}{*}{ P-value } & \multirow{2}{*}{ Sig. } \\
\hline & & & & & & \\
\hline base $0 \mathrm{~min}$ & $\begin{array}{l}\text { Mean } \pm \text { SD } \\
\text { Range }\end{array}$ & $\begin{array}{c}83.00 \pm 5.82 \\
73-91\end{array}$ & $\begin{array}{c}105.60 \pm 7.37 \\
94-120\end{array}$ & -10.759 & 0.001 & HS \\
\hline 5 min & $\begin{array}{l}\text { Mean } \pm \text { SD } \\
\text { Range }\end{array}$ & $\begin{array}{c}69.85 \pm 6.79 \\
60-80\end{array}$ & $\begin{array}{c}105.65 \pm 7.36 \\
95-120\end{array}$ & -15.989 & 0.001 & HS \\
\hline $10 \mathrm{~min}$ & $\begin{array}{l}\text { Mean } \pm \text { SD } \\
\text { Range }\end{array}$ & $\begin{array}{c}64.40 \pm 5.09 \\
55-74\end{array}$ & $\begin{array}{c}103.15 \pm 4.75 \\
95-110\end{array}$ & -24.886 & 0.001 & HS \\
\hline $15 \mathrm{~min}$ & $\begin{array}{l}\text { Mean } \pm \text { SD } \\
\text { Range }\end{array}$ & $\begin{array}{l}63.30 \pm 3.91 \\
57-70\end{array}$ & $\begin{array}{c}103.25 \pm 6.13 \\
90-115\end{array}$ & -24.578 & 0.001 & HS \\
\hline $20 \mathrm{~min}$ & \begin{tabular}{|l|} 
Mean \pm SD \\
Range
\end{tabular} & $\begin{array}{c}65.15 \pm 2.96 \\
60-70 \\
\end{array}$ & $\begin{array}{c}104.00 \pm 5.28 \\
90-110 \\
\end{array}$ & -28.695 & 0.001 & HS \\
\hline $30 \mathrm{~min}$ & $\begin{array}{l}\text { Mean } \pm \text { SD } \\
\text { Range }\end{array}$ & $\begin{array}{c}63.80 \pm 2.69 \\
59-69\end{array}$ & $\begin{array}{c}109.35 \pm 5.96 \\
98-120\end{array}$ & -31.165 & 0.001 & HS \\
\hline 1 hour & $\begin{array}{l}\text { Mean } \pm \text { SD } \\
\text { Range }\end{array}$ & $\begin{array}{c}83.00 \pm 5.82 \\
73-91\end{array}$ & $\begin{array}{c}105.60 \pm 7.37 \\
94-120\end{array}$ & -10.759 & 0.001 & HS \\
\hline 1.30hour & $\begin{array}{l}\text { Mean } \pm \text { SD } \\
\text { Range }\end{array}$ & $\begin{array}{c}88.10 \pm 6.03 \\
80-100 \\
\end{array}$ & $\begin{array}{c}108.60 \pm 6.59 \\
100-120 \\
\end{array}$ & -10.261 & 0.001 & $\mathrm{HS}$ \\
\hline 2 hours & \begin{tabular}{|l|} 
Mean \pm SD \\
Range
\end{tabular} & $\begin{array}{c}88.10 \pm 6.03 \\
80-100\end{array}$ & $\begin{array}{c}108.60 \pm 6.59 \\
100-120 \\
\end{array}$ & -10.261 & 0.001 & HS \\
\hline 4 hours & $\begin{array}{l}\text { Mean } \pm \text { SD } \\
\text { Range }\end{array}$ & $\begin{array}{c}104.2 \pm 9.0 \\
80-100\end{array}$ & $\begin{array}{c}108.60 \pm 6.59 \\
100-120\end{array}$ & 1.764 & 0.086 & NS \\
\hline 6 hours & $\begin{array}{l}\text { Mean } \pm \text { SD } \\
\text { Range }\end{array}$ & $\begin{array}{l}104.5 \pm 7.0 \\
80-100\end{array}$ & $\begin{array}{c}108.60 \pm 6.59 \\
100-120\end{array}$ & 1.907 & 0.064 & NS \\
\hline 12 hours & \begin{tabular}{|l} 
Mean \pm SD \\
Range
\end{tabular} & $\begin{array}{c}105.0 \pm 13.0 \\
80-100\end{array}$ & $\begin{array}{c}108.60 \pm 6.59 \\
100-120\end{array}$ & 1.105 & 0.276 & NS \\
\hline 18 hours & $\begin{array}{l}\text { Mean } \pm \text { SD } \\
\text { Range }\end{array}$ & $\begin{array}{c}106.0 \pm 13 \\
80-100\end{array}$ & $\begin{array}{c}108.60 \pm 6.59 \\
100-120\end{array}$ & 0.798 & 0.430 & NS \\
\hline 24 hours & $\begin{array}{l}\text { Mean } \pm \text { SD } \\
\text { Range }\end{array}$ & $\begin{array}{c}107.2 \pm 13 \\
80-100\end{array}$ & $\begin{array}{c}108.60 \pm 6.59 \\
100-120\end{array}$ & 0.430 & 0.670 & $\mathrm{NS}$ \\
\hline
\end{tabular}

P-value >0.05: Non significant (NS); P-value <0.05: Significant (S); P-value $<0.01$ : highly significant (HS)

$\because$ : Independent t-test

Table (2): Difference between group (S) and group (B) regarding regard oxygen saturation (spo2\%). 
Samir El-Habashy et al.

\begin{tabular}{|c|c|c|c|c|c|c|}
\hline \multirow{2}{*}{\multicolumn{2}{|c|}{$\begin{array}{c}\text { As regard oxygen } \\
\text { saturation }(\text { spo2\%) }\end{array}$}} & \multirow{2}{*}{$\begin{array}{l}\text { Group S } \\
\text { No. }=\mathbf{2 0} \\
\end{array}$} & \multirow{2}{*}{$\begin{array}{l}\text { Group B } \\
\text { No. }=\mathbf{2 0} \\
\end{array}$} & \multirow{2}{*}{$\begin{array}{c}\text { Test } \\
\text { value }\end{array}$} & \multirow{2}{*}{$\begin{array}{c}\mathbf{P}- \\
\text { value }\end{array}$} & \multirow{2}{*}{ Sig } \\
\hline & & & & & & \\
\hline base 0 min & $\begin{array}{l}\text { Mean } \pm \text { SD } \\
\text { Range }\end{array}$ & $\begin{array}{c}98.65 \pm 0.81 \\
97-100 \\
\end{array}$ & $\begin{array}{c}98.65 \pm 0.81 \\
97-100 \\
\end{array}$ & 0.000 & 1.000 & NS \\
\hline $5 \min$ & $\begin{array}{l}\text { Mean } \pm \text { SD } \\
\text { Range }\end{array}$ & $\begin{array}{c}98.65 \pm 0.81 \\
97-100\end{array}$ & $\begin{array}{c}98.65 \pm 0.81 \\
97-100\end{array}$ & 0.000 & 1.000 & NS \\
\hline $10 \mathrm{~min}$ & \begin{tabular}{|l} 
Mean \pm SD \\
Range
\end{tabular} & $\begin{array}{c}98.65 \pm 0.81 \\
97-100 \\
\end{array}$ & $\begin{array}{c}98.65 \pm 0.81 \\
97-100 \\
\end{array}$ & 0.000 & 1.000 & NS \\
\hline $15 \mathrm{~min}$ & $\begin{array}{l}\text { Mean } \pm \text { SD } \\
\text { Range }\end{array}$ & $\begin{array}{c}98.65 \pm 0.81 \\
97-100 \\
\end{array}$ & $\begin{array}{c}98.65 \pm 0.81 \\
97-100 \\
\end{array}$ & 0.000 & 1.000 & NS \\
\hline $20 \mathrm{~min}$ & $\begin{array}{l}\text { Mean } \pm \text { SD } \\
\text { Range }\end{array}$ & $\begin{array}{c}98.65 \pm 0.81 \\
97-100 \\
\end{array}$ & $\begin{array}{c}96.10 \pm 0.79 \\
95-98 \\
\end{array}$ & 10.074 & 0.001 & HS \\
\hline $30 \mathrm{~min}$ & $\begin{array}{l}\text { Mean } \pm \text { SD } \\
\text { Range }\end{array}$ & $\begin{array}{c}98.65 \pm 0.81 \\
97-100 \\
\end{array}$ & $\begin{array}{c}96.10 \pm 0.79 \\
95-98 \\
\end{array}$ & 10.074 & 0.001 & HS \\
\hline 1 hour & $\begin{array}{l}\text { Mean } \pm \text { SD } \\
\text { Range }\end{array}$ & $\begin{array}{c}98.65 \pm 0.81 \\
97-100 \\
\end{array}$ & $\begin{array}{c}94.80 \pm 0.70 \\
94-96 \\
\end{array}$ & 16.092 & 0.001 & HS \\
\hline 1.30hour & $\begin{array}{l}\text { Mean } \pm \text { SD } \\
\text { Range }\end{array}$ & $\begin{array}{c}98.65 \pm 0.81 \\
97-100 \\
\end{array}$ & $\begin{array}{c}94.80 \pm 0.70 \\
94-96 \\
\end{array}$ & 16.092 & 0.001 & HS \\
\hline 2 hours & $\begin{array}{l}\text { Mean } \pm \text { SD } \\
\text { Range }\end{array}$ & $\begin{array}{c}98.60 \pm 0.75 \\
97-100 \\
\end{array}$ & $\begin{array}{c}94.80 \pm 0.70 \\
94-96 \\
\end{array}$ & 16.564 & 0.001 & HS \\
\hline 4 hours & $\begin{array}{l}\text { Mean } \pm \text { SD } \\
\text { Range }\end{array}$ & $\begin{array}{c}98.55 \pm 0.69 \\
97-100 \\
\end{array}$ & $\begin{array}{c}97.0 \pm 3.55 \\
94-96 \\
\end{array}$ & -1.917 & 0.063 & NS \\
\hline 6 hours & $\begin{array}{l}\text { Mean } \pm \text { SD } \\
\text { Range }\end{array}$ & $\begin{array}{c}98.50 \pm 0.61 \\
97-99 \\
\end{array}$ & $\begin{array}{c}97.5 \pm 2.3 \\
95-98 \\
\end{array}$ & -1.879 & 0.068 & NS \\
\hline 12 hours & $\begin{array}{l}\text { Mean } \pm \text { SD } \\
\text { Range }\end{array}$ & $\begin{array}{c}98.50 \pm 0.61 \\
97-99 \\
\end{array}$ & $\begin{array}{c}97.8 \pm 1.5 \\
95-98 \\
\end{array}$ & -1.933 & 0.061 & NS \\
\hline 18 hours & $\begin{array}{l}\text { Mean } \pm \text { SD } \\
\text { Range } \\
\end{array}$ & $\begin{array}{c}98.50 \pm 0.61 \\
97-99 \\
\end{array}$ & $\begin{array}{c}97.8 \pm 1.5 \\
95-98 \\
\end{array}$ & -1.933 & 0.061 & NS \\
\hline 24 hours & $\begin{array}{l}\text { Mean } \pm \text { SD } \\
\text { Range }\end{array}$ & $\begin{array}{c}98.50 \pm 0.61 \\
97-99\end{array}$ & $\begin{array}{c}97.8 \pm 1.5 \\
95-98 \\
\end{array}$ & -1.933 & 0.061 & NS \\
\hline
\end{tabular}

P-value >0.05: Non significant (NS); P-value <0.05: Significant (S); P-value $<0.01$ : highly significant (HS)

$\bullet$ Independent t-test

Table (3): Difference between group (S) and group (B) regarding heart rate 


\begin{tabular}{|c|c|c|c|c|c|c|}
\hline \multirow{2}{*}{\multicolumn{2}{|c|}{ As regard heart rate }} & \multirow{2}{*}{$\begin{array}{l}\text { Group S } \\
\text { No. }=\mathbf{2 0} \\
\end{array}$} & \multirow{2}{*}{$\begin{array}{l}\text { Group B } \\
\text { No. }=\mathbf{2 0} \\
\end{array}$} & \multirow{2}{*}{$\begin{array}{c}\text { Test } \\
\text { value• }\end{array}$} & \multirow{2}{*}{\begin{tabular}{|c|}
$\mathbf{P}-$ \\
value
\end{tabular}} & \multirow{2}{*}{ Sig. } \\
\hline & & & & & & \\
\hline base $0 \mathrm{~min}$ & $\begin{array}{l}\text { Mean } \pm \text { SD } \\
\text { Range }\end{array}$ & $\begin{array}{c}79.10 \pm 3.0 \\
77-80 \\
\end{array}$ & $\begin{array}{c}78.0 \pm 0.85 \\
77-80 \\
\end{array}$ & -1.578 & 0.123 & NS \\
\hline $5 \mathrm{~min}$ & $\begin{array}{l}\text { Mean } \pm \text { SD } \\
\text { Range }\end{array}$ & $\begin{array}{c}88 \pm 4.3 \\
77-82\end{array}$ & $\begin{array}{c}86 \pm 1.39 \\
77-82\end{array}$ & -1.979 & 0.055 & NS \\
\hline $10 \mathrm{~min}$ & $\begin{array}{l}\text { Mean } \pm \text { SD } \\
\text { Range }\end{array}$ & $\begin{array}{c}90.0 \pm 2.3 \\
77-80 \\
\end{array}$ & $\begin{array}{c}89 \pm 0.85 \\
77-80 \\
\end{array}$ & -1.824 & 0.076 & NS \\
\hline $15 \mathrm{~min}$ & $\begin{array}{l}\text { Mean } \pm \text { SD } \\
\text { Range }\end{array}$ & $\begin{array}{c}91.0 \pm 1.39 \\
77-82 \\
\end{array}$ & $\begin{array}{l}90.0 \pm 2 \\
77-82 \\
\end{array}$ & -1.836 & 0.074 & NS \\
\hline $20 \mathrm{~min}$ & $\begin{array}{l}\text { Mean } \pm \text { SD } \\
\text { Range }\end{array}$ & $\begin{array}{c}91.0 \pm 1.39 \\
77-82 \\
\end{array}$ & $\begin{array}{l}90.0 \pm 2 \\
77-82 \\
\end{array}$ & -1.836 & 0.074 & NS \\
\hline $30 \mathrm{~min}$ & $\begin{array}{l}\text { Mean } \pm \text { SD } \\
\text { Range }\end{array}$ & $\begin{array}{c}91.0 \pm 1.39 \\
77-82 \\
\end{array}$ & $\begin{array}{l}90.0 \pm 2 \\
77-82 \\
\end{array}$ & -1.836 & 0.074 & NS \\
\hline 1 hour & $\begin{array}{l}\text { Mean } \pm \text { SD } \\
\text { Range }\end{array}$ & $\begin{array}{c}93.0 \pm 0.85 \\
77-80 \\
\end{array}$ & $\begin{array}{c}92.8 \pm 1.5 \\
77-80 \\
\end{array}$ & -0.519 & 0.607 & NS \\
\hline 1.30hour & $\begin{array}{l}\text { Mean } \pm \text { SD } \\
\text { Range }\end{array}$ & $\begin{array}{c}93.0 \pm 0.85 \\
77-80 \\
\end{array}$ & $\begin{array}{c}92.8 \pm 1.5 \\
77-80 \\
\end{array}$ & -0.519 & 0.607 & NS \\
\hline 2 hours & $\begin{array}{l}\text { Mean } \pm \text { SD } \\
\text { Range }\end{array}$ & $\begin{array}{c}92.8 \pm 0.50 \\
77-80 \\
\end{array}$ & $\begin{array}{c}91.9 \pm 2.1 \\
77-80 \\
\end{array}$ & -1.865 & 0.070 & NS \\
\hline 4 hours & $\begin{array}{l}\text { Mean } \pm \text { SD } \\
\text { Range }\end{array}$ & $\begin{array}{c}85.0 \pm 1.39 \\
77-82 \\
\end{array}$ & $\begin{array}{c}84.3 \pm 1.39 \\
77-82 \\
\end{array}$ & -1.593 & 0.120 & NS \\
\hline 6 hours & $\begin{array}{l}\text { Mean } \pm \text { SD } \\
\text { Range }\end{array}$ & $\begin{array}{c}80.0 \pm 0.85 \\
77-80 \\
\end{array}$ & $\begin{array}{c}79.90 \pm 0.85 \\
77-80 \\
\end{array}$ & -0.372 & 0.712 & NS \\
\hline 12 hours & $\begin{array}{l}\text { Mean } \pm \text { SD } \\
\text { Range }\end{array}$ & $\begin{array}{c}80.9 \pm 1.4 \\
77-82 \\
\end{array}$ & $\begin{array}{c}79.9 \pm 2.0 \\
77-82 \\
\end{array}$ & -1.832 & 0.075 & NS \\
\hline 18 hours & $\begin{array}{l}\text { Mean } \pm \text { SD } \\
\text { Range }\end{array}$ & $\begin{array}{c}80.0 \pm 0.85 \\
77-80 \\
\end{array}$ & $\begin{array}{c}79.50 \pm 0.85 \\
77-80 \\
\end{array}$ & -1.860 & 0.071 & NS \\
\hline 24 hours & $\begin{array}{l}\text { Mean } \pm \text { SD } \\
\text { Range }\end{array}$ & $\begin{array}{c}81.1 \pm 1.39 \\
77-82\end{array}$ & $\begin{array}{c}80.65 \pm 1.39 \\
77-82 \\
\end{array}$ & -1.024 & 0.312 & NS \\
\hline
\end{tabular}

P-value >0.05: Non significant (NS); P-value <0.05: Significant (S); P-value $<0.01$ : highly significant (HS)

$\bullet:$ Independent t-test

Table (4): Difference between group(S) and group (B) regarding respiratory rate 


\begin{tabular}{|c|c|c|c|c|c|c|}
\hline \multirow{2}{*}{\multicolumn{2}{|c|}{ As regard respiratory rate }} & \multirow{2}{*}{$\begin{array}{c}\text { Group S } \\
\text { No. }=20\end{array}$} & \multirow{2}{*}{$\begin{array}{c}\text { Group B } \\
\text { No. }=20\end{array}$} & \multirow{2}{*}{$\begin{array}{c}\text { Test } \\
\text { value• }\end{array}$} & \multirow{2}{*}{\begin{tabular}{|c|}
$\mathbf{P}-$ \\
value
\end{tabular}} & \multirow{2}{*}{ Sig. } \\
\hline & & & & & & \\
\hline base $0 \mathrm{~min}$ & $\begin{array}{l}\text { Mean } \pm \text { SD } \\
\text { Range }\end{array}$ & $\begin{array}{c}17.0 \pm 0.67 \\
16-18\end{array}$ & $\begin{array}{c}16.65 \pm 0.67 \\
16-18\end{array}$ & -1.652 & 0.107 & NS \\
\hline $5 \mathrm{~min}$ & \begin{tabular}{|l} 
Mean \pm SD \\
Range
\end{tabular} & $\begin{array}{c}17.5 \pm 0.60 \\
17-19\end{array}$ & $\begin{array}{c}17.55 \pm 0.60 \\
17-19\end{array}$ & 0.264 & 0.794 & NS \\
\hline $10 \mathrm{~min}$ & $\begin{array}{l}\text { Mean } \pm \text { SD } \\
\text { Range }\end{array}$ & $\begin{array}{c}17.2 \pm 1.2 \\
16-18\end{array}$ & $\begin{array}{c}16.65 \pm 0.67 \\
16-18\end{array}$ & -1.790 & 0.082 & NS \\
\hline $15 \mathrm{~min}$ & $\begin{array}{l}\text { Mean } \pm \text { SD } \\
\text { Range }\end{array}$ & $\begin{array}{l}18.0 \pm 1.2 \\
17-19\end{array}$ & $\begin{array}{c}17.55 \pm 0.60 \\
17-19\end{array}$ & -1.500 & 0.142 & NS \\
\hline $20 \mathrm{~min}$ & $\begin{array}{l}\text { Mean } \pm \text { SD } \\
\text { Range }\end{array}$ & $\begin{array}{c}17.0 \pm 1.1 \\
16-18\end{array}$ & $\begin{array}{c}16.65 \pm 0.67 \\
16-18\end{array}$ & -1.215 & 0.232 & NS \\
\hline $30 \mathrm{~min}$ & $\begin{array}{l}\text { Mean } \pm \text { SD } \\
\text { Range }\end{array}$ & $\begin{array}{c}18.0 \pm 0.99 \\
17-19\end{array}$ & $\begin{array}{c}17.55 \pm 0.60 \\
17-19\end{array}$ & -1.738 & 0.090 & NS \\
\hline 1hour & $\begin{array}{l}\text { Mean } \pm \text { SD } \\
\text { Range }\end{array}$ & $\begin{array}{c}17.2 \pm 1.3 \\
16-18\end{array}$ & $\begin{array}{c}16.65 \pm 0.67 \\
16-18\end{array}$ & -1.682 & 0.101 & NS \\
\hline 1.30hour & $\begin{array}{l}\text { Mean } \pm \text { SD } \\
\text { Range }\end{array}$ & $\begin{array}{c}18.1 \pm 1.3 \\
17-19\end{array}$ & $\begin{array}{c}17.55 \pm 0.60 \\
17-19\end{array}$ & -1.718 & 0.094 & NS \\
\hline 2 hours & $\begin{array}{l}\text { Mean } \pm \text { SD } \\
\text { Range }\end{array}$ & $\begin{array}{c}17.0 \pm 1.1 \\
16-18\end{array}$ & $\begin{array}{c}16.65 \pm 0.67 \\
16-18\end{array}$ & -1.300 & 0.201 & NS \\
\hline 4 hours & $\begin{array}{l}\text { Mean } \pm \text { SD } \\
\text { Range }\end{array}$ & $\begin{array}{c}18.1 \pm 1.8 \\
17-19\end{array}$ & $\begin{array}{c}17.55 \pm 0.60 \\
17-19\end{array}$ & -1.296 & 0.203 & NS \\
\hline 6 hours & $\begin{array}{l}\text { Mean } \pm \text { SD } \\
\text { Range }\end{array}$ & $\begin{array}{c}17.1 \pm 1.2 \\
16-18\end{array}$ & $\begin{array}{c}16.65 \pm 0.67 \\
16-18\end{array}$ & -1.464 & 0.151 & NS \\
\hline 12 hours & \begin{tabular}{|l} 
Mean \pm SD \\
Range
\end{tabular} & $\begin{array}{c}18.2 \pm 1.50 \\
17-19\end{array}$ & $\begin{array}{c}17.55 \pm 0.60 \\
17-19\end{array}$ & -1.799 & 0.080 & NS \\
\hline 18 hours & $\begin{array}{l}\text { Mean } \pm \text { SD } \\
\text { Range }\end{array}$ & $\begin{array}{c}17.0 \pm 1.2 \\
16-18\end{array}$ & $\begin{array}{c}16.65 \pm 0.67 \\
16-18\end{array}$ & -1.139 & 0.262 & NS \\
\hline 24 hours & $\begin{array}{l}\text { Mean } \pm \text { SD } \\
\text { Range }\end{array}$ & $\begin{array}{c}18.0 \pm 1.30 \\
17-19\end{array}$ & $\begin{array}{c}17.55 \pm 0.60 \\
17-19\end{array}$ & -1.406 & 0.168 & NS \\
\hline
\end{tabular}

P-value >0.05: Non significant (NS); P-value <0.05: Significant (S); P-value< 0.01: highly significant (HS)

$\bullet$ Independent t-test

Table (5): Difference between group (S) and group (B) regarding preparation time (PT) and surgical anesthesia time (SAT)

\begin{tabular}{|l|c|c|c|c|c|}
\hline & Group s & Group B & \multirow{2}{*}{ Test value• } & P-value & Sig. \\
\cline { 2 - 3 } & No. $=\mathbf{2 0}$ & No. = 20 & & & \\
\hline Preparation time (min) & $4.85 \pm 0.58$ & $25.2 \pm 0.61$ & 108.121 & 0.001 & HS \\
\hline $\begin{array}{l}\text { Surgical anesthesia time } \\
\text { (min) }\end{array}$ & $9.88 \pm 2$ & $16.88 \pm 1.6$ & 12.223 & 0.001 & HS \\
\hline
\end{tabular}

P-value > 0.05: Non significant; P-value < 0.05: Significant; P-value < 0.01: Highly significant

$\bullet:$ Independent t-test

Table (6): Difference between group (S) and group (B) regarding degree of motor block 


\begin{tabular}{|c|c|c|c|c|c|}
\hline & Group S & Group B & \multirow{2}{*}{ Test value } & \multirow{2}{*}{ P-value } & \multirow{2}{*}{ Sig. } \\
\hline & No. $=20$ & No. $=20$ & & & \\
\hline Onset of motor block (min) & $6.52 \pm 2$ & $23.8 \pm 6$ & $12.219 \bullet$ & 0.000 & $\mathrm{HS}$ \\
\hline $\begin{array}{l}\text { Grade of motor block after } 1 \text { hour } \\
\text { I } \\
\text { II } \\
\text { III } \\
\text { IV }\end{array}$ & $\begin{array}{l}0(0.0 \%) \\
0(0.0 \%) \\
2(10.0 \%) \\
18(90 \%)\end{array}$ & $\begin{array}{c}0(0.0 \%) \\
0(0.0 \%) \\
13(65.0 \%) \\
7(35.0 \%)\end{array}$ & $12.907 *$ & 0.001 & HS \\
\hline $\begin{array}{l}\text { Grade of motor block after } 4 \text { hours } \\
\text { I } \\
\text { II } \\
\text { III } \\
\text { IV }\end{array}$ & $\begin{array}{c}20(100.0 \%) \\
0(0.0 \%) \\
0(0.0 \%) \\
0(0.0 \%) \\
\end{array}$ & $\begin{array}{c}6(30.0 \%) \\
3(15.0 \%) \\
11(55.0 \%) \\
0(0.0 \%) \\
\end{array}$ & $21.538 *$ & 0.001 & HS \\
\hline $\begin{array}{l}\text { Intra operative free movement of knee joint } \\
\text { Excellent } \\
\text { Sufficient } \\
\text { Insufficient }\end{array}$ & $\begin{array}{c}15(75.0 \%) \\
5(25.0 \%) \\
0(0.0 \%)\end{array}$ & $\begin{array}{c}18(90.0 \%) \\
2(10.0 \%) \\
0(0.0 \%)\end{array}$ & $1.558 * *$ & 0.459 & $\mathrm{NS}$ \\
\hline
\end{tabular}

P-value > 0.05: Non significant; P-value < 0.05: Significant; P-value < 0.01: Highly significant

$*$ : Chi-square test; $\bullet$ : Independent t-test

Table (7): Description of the Bromage score. Grade Criteria Degree of Block

\begin{tabular}{|c|c|c|}
\hline Grade & Criteria & Degree of block \\
\hline I & Free movement of leg, feet & N.T (0) \\
\hline II & Just able to flex knee with free movement of feet & Partial (33\%) \\
\hline III & Unable to flex knee but free movement of feet & Almost complete (66\%) \\
\hline IV & Unable to move leg or feet & Complete (100\%) \\
\hline
\end{tabular}

Table (8): Difference between group (S) and group (B) regarding visual analogue score (VAS)

\begin{tabular}{|c|c|c|c|c|c|}
\hline & Group s & Group B & \multirow{2}{*}{ Test value• } & \multirow{2}{*}{$P$-value } & \multirow{2}{*}{ Sig. } \\
\hline & No. $=20$ & No. $=20$ & & & \\
\hline \multicolumn{6}{|c|}{ VAS score } \\
\hline Baseline & $1.5 \pm 0.35$ & $1.5 \pm 0.45$ & 0.000 & 1.000 & NS \\
\hline $15 \mathrm{~min}$ & $1 \pm 0.2$ & $1 \pm 0.28$ & 0.000 & 1.000 & NS \\
\hline $30 \mathrm{~min}$ & $1 \pm 0.23$ & $1 \pm 0.37$ & 0.000 & 1.000 & NS \\
\hline $45 \mathrm{~min}$ & $1 \pm 0.27$ & $1 \pm 0.35$ & 0.000 & 1.000 & NS \\
\hline $60 \mathrm{~min}$ & $1 \pm 0.3$ & $1 \pm 0.39$ & 0.000 & 1.000 & NS \\
\hline $75 \mathrm{~min}$ & $1 \pm 0.25$ & $1 \pm 0.32$ & 0.000 & 1.000 & NS \\
\hline $90 \mathrm{~min}$ & $1 \pm 0.32$ & $1 \pm 0.22$ & 0.000 & 1.000 & NS \\
\hline $105 \mathrm{~min}$ & $1 \pm 0.34$ & $1 \pm 0.24$ & 0.000 & 1.000 & NS \\
\hline $120 \mathrm{~min}$ & $1 \pm 0.36$ & $1 \pm 0.25$ & 0.000 & 1.000 & NS \\
\hline $4 \mathrm{hr}$ & $3 \pm 0.4$ & $2 \pm 0.34$ & -8.519 & 0.001 & HS \\
\hline $6 \mathrm{hr}$ & $3 \pm 0.46$ & $2 \pm 0.3$ & -8.143 & 0.001 & $\mathrm{HS}$ \\
\hline $10 \mathrm{hr}$ & $3 \pm 0.5$ & $1.5 \pm 0.38$ & -10.682 & 0.001 & HS \\
\hline $18 \mathrm{hr}$ & $3 \pm 0.55$ & $2 \pm 0.55$ & -5.750 & 0.001 & $\mathrm{HS}$ \\
\hline $24 \mathrm{hr}$ & $3 \pm 0.52$ & $2 \pm 0.52$ & -6.081 & 0.001 & $\mathrm{HS}$ \\
\hline
\end{tabular}

P-value > 0.05: Non significant; P-value < 0.05: Significant; P-value < 0.01: Highly significant

*: Chi-square test; $\bullet:$ Independent t-test

Table (9): Difference between group (S) and group (B) regarding total anesthesia time (TAT) and the time of first postoperative analgesia. 


\begin{tabular}{|c|c|c|c|c|c|}
\hline & Group S & Group B & \multirow{2}{*}{ Test value• } & \multirow{2}{*}{$P$-value } & \multirow{2}{*}{ Sig. } \\
\hline & No. $=20$ & No. $=20$ & & & \\
\hline $\begin{array}{c}\text { Total anesthesia time } \\
(\mathrm{min})\end{array}$ & $160 \pm 20$ & $340.8 \pm 36.69$ & 19.350 & 0.001 & HS \\
\hline $\begin{array}{c}\text { Time of } 1^{\text {st }} \text { post operative } \\
\text { analgesia (min) }\end{array}$ & $175.75 \pm 17.2$ & $360.88 \pm 21.2$ & 30.327 & 0.001 & HS \\
\hline
\end{tabular}

P-value > 0.05: Non significant; P-value < 0.05: Significant; P-value < 0.01: Highly significant

$\because$ Independent t-test

Table (10): Difference between group (S) and group (B) regarding intraoperative complications

\begin{tabular}{|l|c|c|c|c|c|}
\hline \multirow{2}{*}{ Intraoperative complications } & Group S & Group B & \multirow{2}{*}{ Test value* } & P-value & \multirow{2}{*}{ Sig. } \\
\cline { 2 - 4 } & No. (\%) & No. (\%) & & & \\
\hline No & $11(55.0 \%)$ & $20(100.0 \%)$ & & & \\
\cline { 1 - 4 } Hypotension & $5(25.0 \%)$ & $0(0.0 \%)$ & \multirow{2}{*}{11.613} & 0.009 & HS \\
\hline Nausea, vomiting & $3(15.0 \%)$ & $0(0.0 \%)$ & & & \\
\hline
\end{tabular}

P-value > 0.05: Non significant; P-value < 0.05: Significant; P-value < 0.01: Highly significant

*: Chi-square test

Table (11): Difference between group (S) and group (B) regarding postoperative complications

\begin{tabular}{|c|c|c|c|c|c|}
\hline \multirow{2}{*}{$\begin{array}{l}\text { Postoperative } \\
\text { complications }\end{array}$} & Group S & Group B & \multirow{2}{*}{ Test value* } & \multirow{2}{*}{ P-value } & \multirow{2}{*}{ Sig. } \\
\hline & No. $(\%)$ & No. $(\%)$ & & & \\
\hline No & $10(50.0 \%)$ & $20(100.0 \%)$ & \multirow{7}{*}{13.333} & \multirow{7}{*}{0.009} & \multirow{7}{*}{ HS } \\
\hline Headache & $4(20.0 \%)$ & $0(0.0 \%)$ & & & \\
\hline Nausea\& vomiting & $3(15.0 \%)$ & $0(0.0 \%)$ & & & \\
\hline Backache & $2(10.0 \%)$ & $0(0.0 \%)$ & & & \\
\hline Retention of urine & $1(5.0 \%)$ & $0(0.0 \%)$ & & & \\
\hline Itching\& pruritus & $0(0.0 \%)$ & $0(0.0 \%)$ & & & \\
\hline Neurological sequelae & $0(0.0 \%)$ & $0(0.0 \%)$ & & & \\
\hline
\end{tabular}

P-value > 0.05: Non significant; P-value < 0.05: Significant; P-value < 0.01: Highly significant

*: Chi-square test

Table (12): Difference between group (S) \& group (B) regarding patient satisfaction

\begin{tabular}{|c|c|c|c|c|c|}
\hline \multirow{2}{*}{ Patient satisfaction } & Group S & Group B & \multirow{2}{*}{ Test value* } & \multirow{2}{*}{ P-value } & \multirow{2}{*}{ Sig. } \\
\hline & No. $(\%)$ & No. $(\%)$ & & & \\
\hline Perfect & $15(75.0 \%)$ & $6(30.0 \%)$ & \multirow{4}{*}{8.622} & \multirow{4}{*}{0.035} & \multirow{4}{*}{$\mathrm{S}$} \\
\hline Acceptable & $4(20.0 \%)$ & $13(65.0 \%)$ & & & \\
\hline Poor & $1(5.0 \%)$ & $1(5.0 \%)$ & & & \\
\hline Unsuccessful & $0(0.0 \%)$ & $0(0.0 \%)$ & & & \\
\hline
\end{tabular}

P-value > 0.05: Non significant; P-value < 0.05: Significant; P-value < 0.01: Highly significant

*: Chi-square test

Table (13): Difference between group (S) \& group (B) regarding surgeon satisfaction

\begin{tabular}{|l|c|c|c|c|c|}
\hline \multirow{2}{*}{ Surgeon satisfaction } & Group S & Group B & \multirow{2}{*}{ Test value* } & \multirow{2}{*}{ P-value } & \multirow{2}{*}{ Sig. } \\
\cline { 2 - 3 } & No. (\%) & No. (\%) & & & \\
\hline Perfect & $11(55.0 \%)$ & $9(45.0 \%)$ & & & \multirow{2}{*}{0.400} \\
Acceptable & $9(45.0 \%)$ & $11(55.0 \%)$ & 0.527 & NS \\
\hline Poor & $0(0.0 \%)$ & $0(0.0 \%)$ & & & \\
\hline Unsuccessful & $0(0.0 \%)$ & $0(0.0 \%)$ & & & \\
\hline
\end{tabular}

P-value > 0.05: Non significant; P-value < 0.05: Significant; P-value < 0.01: Highly significant

*: Chi-square test

DISCUSSION 
Ambulatory orthopaedic procedures have become increasingly common due to the effectiveness of the anesthesia techniques that facilitate rapid and safe discharge ${ }^{(l)}$.

Both peripheral nerve blocks and spinal anesthesia provide sufficient anesthesia, better postoperative analgesia, and satisfaction than general anesthesia, in addition to being minimally invasive and using fewer resources (2).

Spinal anesthesia is particularly preferred by patients undergoing unilateral lower limb surgery due to the fact that only the desired region undergoes nerve blockade, which results in early mobilization and good patient satisfaction ${ }^{(3)}$.

The selection of the anesthesia technique and the anesthetic agents are important as the adverse events and complications affect discharge time, ultrasound fascia iliaca compartment and sciatic nerve block appears to be a good alternative for spinal anesthesia in ambulatory knee surgery ${ }^{(9)}$.

In the present study, sufficient block could not be obtained in two patients in block group, they shifted to general anesthesia and excluded from the evaluation, and we replaced them by two other patients.

Also in the present study there was no statistically difference in the demographic data between the two groups regarding age, sex, weight, height, ASA, and duration of surgery.

These results coincide with the results reached by Davarci and his colleagues who compared spinal anesthesia with sciatic and femoral nerve block on 40 patients scheduled to undergo ambulatory arthroscopic knee surgery. They found no statistically difference between two groups regarding demographic and hemodynamic data at variable times intraoperatively ${ }^{(10)}$.

Also no statistically significant difference was found among the two groups as regard measurement of heart rate and respiratory rate except there was nonsignificant increase in heart rate in group (S) than group (B) at first 120 minutes due to reflex tachycardia of ephedrine which was given to correct hypotension.

But there was significant statistically difference in mean arterial blood pressure at first 120 minutes and oxygen saturation measurement from 20 to 120 minutes (due to using sedoanalgesia from the start) intraoperatively.
These results coincide with the results reached by Akkaya and his colleagues performed a study on ultrasound-guided femoral and sciatic nerve blocks combined with sedoanalgesia versus spinal anesthesia. Sedoanalgesia was given with $1 \mathrm{mg}$ of midazolam, $50 \mu \mathrm{g}$ fentanyl, and $10-15 \mathrm{mg}$ ketamine from the start ${ }^{(11)}$.

Mean arterial pressure was significantly lower in the spinal group this may be attributed to the large dose of local anesthetic (4 ml) during spinal anesthesia. oxygen saturation was decreased in the block group up to $92 \%$, this may be due to using sedoanalgesia from the start ${ }^{(I I)}$.

But in the present study there was statistically difference in haemodynamic data as mean arterial blood pressure measurement and oxgen saturation.

Also in the present study we used ultrasound guided fascia iliaca compartment block instead femoral nerve block.

In the present study the mean preparation time (PT) was $5 \mathrm{~min}$. in group S, but then the patients were required to wait in the lateral decubitus position on the operated side for $5 \mathrm{~min}$; so the mean total time before the initiation of surgery was $10 \mathrm{~min}$. In group B, the mean PT for the fascia iliaca compartment and sciatic nerve block was 15 min followed by a mean of 25 min before the nerve blockade was achieved; thus the mean total time before the initiation of surgery was $40 \mathrm{~min}$. When the two groups were compared, there was a 3o-min delay prior to surgery in the block group which is statistically significant.

Davarci and his colleagues found that there was a 7-min delay prior to surgery in the USFB group which is lower than the present study by $23 \mathrm{~min}$, they did not block (FIC) which may be the cause ${ }^{(10)}$.

In the present study there was no significant difference between the two groups regarding intraoperative free movement of the knee joint by surgeon. This was also the case with Davarciland his colleagues; they also did not find any significant difference between the two groups ${ }^{(10)}$.

In the present study, it was observed a statistically significant difference between the two groups as regard the grades of motor block where group $\mathrm{S}$ showed more solid motor block than group B after 1 hour, whereas it showed full recovery from motor block after 4 hours, on 
the other hand in group B 11 patients still had residual motor block grade III.

These results coincide with that concluded by Davarci and his colleagues. They found that the difference between the two groups in terms of motor blockade was significantly higher in spinal group after 1 hour, whereas after $4 \mathrm{hrs}$, motor block was higher in block group where 14 patients still had grade II motor blockade, while none of the patients in spinal group had motor blockade at the same time-point ${ }^{(10)}$.

In the present study SAT was significantly lower in group $\mathrm{S}$ (9 $\mathrm{min})$ compared to group B (17 min).

These results coincide with the results

found by Davarcil and his colleagues, they found that spinal group had significantly shorter delay time ( 8 minutes) before the beginning of surgery compared to (18 minutes) in the combined sciatic-femoral block group ${ }^{(10)}$.

In the present study, it was noticed that significantly longer total anesthesia time (TAT) in group B when compared with group $\mathrm{S}$ which was also the case with Davarcil and his colleagues $^{(10)}$.

The presence of sufficient postoperative analgesia is essential to facilitate rehabilitation after knee arthroplasty. This was noted in the present study as group (B) had significantly longer time to first analgesia than group (S). This was obvious when additional analgesia was needed after a mean of $175 \mathrm{~min}$. in group (S), while it was needed after $360 \mathrm{~min}$. in group (B).

These results coincide with the results reached by Spasiano and his colleagues, where they found that time to first analgesia was significantly longer in block group than spinal group. Only one patient per group required additional analgesia after $230 \mathrm{~min}$. in the spinal group compared with SFNB group after 310 min. ${ }^{(12)}$.

In the present study, regarding $(10-\mathrm{cm})$ VAS, the group (B) provided significantly more effective analgesia than group (S) at 4, 6, 10, 18 and 24 hours postoperatively, which was also the case with Davarci and his colleagues ${ }^{(10)}$.

In the present study, urinary retention was recorded in group (S) more than group (B).

Davarcil and his colleagues agreed with our study and found that time-to-first spontaneous urination was significantly longer in the spinal group despite of the rapid resolution of the nerve block group ${ }^{(10)}$.

In the present study both patient and surgeon satisfactions were acceptable.

But patient satisfaction was higher in group (S) than group (B). On other hand no difference between the two groups in surgeon satisfaction.

These results coincide with the results reached by Akkaya and his collegues, they found that patient satisfaction differed significantly and the overall median patient satisfaction value in spinal group and block group were $3: 2$ respectively ${ }^{(I I)}$.

\section{CONCLUSION}

In conclusion, the preferential selection and successful use of anesthesia techniques are based on not only having a short preparation time and rapid onset of action, but also on providing lower rate of complications and adverse events, a longer duration of analgesia, good patient satisfaction and optimal condition for patient discharge compared with other available agents.

It can be possible to achieve SAT and PT with ultrasound-guided combined sciaticfascia iliaca compartment block (FICB) as those achieved with spinal anesthesia in patients undergoing surgical procedures of the lower extremities by increasing the experience of the anesthesia team of using ultrasound guidance.

Although ultrasound guided combined fascia iliaca compartment and sciatic nerve block having longer PT and SAT but also have minimal effect on hemodynamics, longer duration of analgesia and motor block, lower rate of complications and adverse events, and optimal conditions for patient discharge compared with spinal anesthesia.

Taking these current findings into consideration, ultrasound guided combined fascia iliaca compartment and sciatic nerve block appears to be a good alternative for spinal anesthesia in knee arthroplasty especially for obese and hemodynamically unstable patients and also it needs further studies on large number of patients.

\section{REFERENCES}

1. Casati A, Cappelleri G, Aldegheri G et al. (2004): Total intravenous anesthesia, spinal anesthesia or combined sciaticfemoral nerve block for outpatient knee 
arthroscopy. Minerva Anesthesiologica, 70(4):493-502.

2. Montes FR, Zarate E, Grueso $\mathbf{R}$ et al. (2008): Comparison of spinal anesthesia with combined sciatic-femoral nerve block for outpatient knee arthroscopy, Journal of Clinical Anesthesia, 20(2):415-420.

3. Borghi B and Wulf $\mathbf{H}$ (2010): Advantages of unilateral spinal anesthesia. Anesthesiology and Intensive Critical Care Medicine, 45 (3): 182-187.

4. Namba RS, Paxton L, Fithian DC et al. (2005): Obesity and perioperative morbidity in total hip and total knee arthroplasty patients. J Arthroplasty, 20(7): 46-50.

5. Jeon YT (2012): Peripheral nerve block for anesthesia in patients having knee arthroplasty. Korean J Anesthesiol., 62(1): 403-4.

6. Goyal M, Taxak $S$ and Goel $M$ (2011): Continuous Spinal Anesthesia in a High Risk Elderly Patient Using Epidural Set.J.Anesth clin pharmacol., 27(1):129-145.

7. Koscielniak-Nielsen ZJ and Dahl JB (2012): Ultrasound-guided peripheral nerve blockade of the upper extremity. Curr Opin Anaesthesiol., 25: 253-9.
8. O'Donnell BD and Iohom G (2008): Regional anesthesia techniques for ambulatory orthopedic surgery, Current Opinion in Anesthesiology, 21(8):723728.

9. Mulroy M (2002): Systemic toxicity and cardiotoxicity from local anesthtics: incidence and preventive measures. Reg. Anesth. Pain Med., 27(6):556- 561.

10. Davarcil I, Tuzcul K, Karcioglu1 M et al. (2013): Comparison between ultrasoundguided sciatic-femoral nerve block and unilateral spinal anesthesia for outpatient knee arthroscopy. Journal of International Medical Research, 1-9.

11. Akkaya A, Tekelioglu UY, Demirhan A et al. (2014): Ultrasound-guided femoral and sciatic nerve blocks combined with sedoanalgesia versus spinal anesthesia in total knee arthroplasty, Korean Society of Anesthesiologists, 67(1):11-23.

12. Spasiano A, Flore I, Pesamosca A et al. (2007): Comparison between spinal anesthesia and sciatic-femoral block for arthroscopic knee surgery. Minerva Anestesiol., 73(1):13-21. 